\title{
Towards a Continuous Record of the Sky
}

\author{
Robert J. Nemiroff ${ }^{1} \&$ J. Bruce Rafert ${ }^{2}$ \\ Department of Physics, Michigan Technological University, Houghton, MI 49931
}

Received 1998 Sep 29; $\quad$ accepted 1999 Apr 26

To appear in: Publications of the Astronomical Society of the Pacific

\footnotetext{
${ }^{1}$ nemiroff@mtu.edu

2 jbrafert@mtu.edu
} 


\begin{abstract}
It is currently feasible to start a continuous digital record of the entire sky sensitive to any visual magnitude brighter than 15 each night. Such a record could be created with a modest array of small telescopes, which collectively generate no more than a few Gigabytes of data daily. Alternatively, a few small telescopes could continually re-point to scan and record the entire sky down to any visual magnitude brighter than 15 with a recurrence epoch of at most a few weeks, again always generating less than one Gigabyte of data each night. These estimates derive from CCD ability and budgets typical of university research projects. As a prototype, we have developed and are utilizing an inexpensive single-telescope system that obtains optical data from about 1500 square degrees. We discuss the general case of creating and storing data from a both an epochal survey, where a small number of telescopes continually scan the sky, and a continuous survey, composed of a constellation of telescopes dedicated each continually inspect a designated section of the sky. We compute specific limitations of canonical surveys in visible light, and estimate that all-sky continuous visual light surveys could be sensitive to magnitude 20 in a single night by about 2010. Possible scientific returns of continuous and epochal sky surveys include continued monitoring of most known variable stars, establishing case histories for variables of future interest, uncovering new forms of stellar variability, discovering the brightest cases of microlensing, discovering new novae and supernovae, discovering new counterparts to gamma-ray bursts, monitoring known Solar System objects, discovering new Solar System objects, and discovering objects that might strike the Earth.
\end{abstract}

Subject headings: stars: variables: general — instrumentation: detectors — 
techniques: photometric — telescopes — surveys 


\section{Introduction}

Astronomers have monitored the sky at least as long as history has been recorded. Only recently, however, has it become possible to conveniently store such information. Sky-monitoring ability is developing along fronts in energy, angle, brightness, and time. The energy frontier can be divided into the energy band of observation and the energy resolution of observation. Similarly, the angular frontier can be divided into the angular band of sky observed, and the angular resolution of observation. Lastly, the temporal frontier can be divided into the length of time an object is observed, and the temporal resolution of the observation. The minimum brightness of observation is a convolution of many of the above parameters mixed with parameters that define the telescope and detector being used, but for convenience will be considered a separate quantity here. Each front carries with it different scientific goals and technological obstacles.

Major strides have been made recently in the creation of static maps of the sky over energy bands across the electromagnetic spectrum. A few notable examples include those in the gamma-ray (EGRET; Fichtel 1996), the X-ray (HEAO; Wood et al. 1984); the ultraviolet (EUVE; Antia 1993), the optical (Second Palomar Sky Survey, Reid et al. 1991;

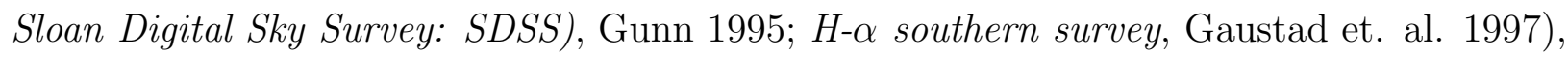
the infrared (Two Micro All Sky Survey: 2MASS; Skrutskie et al. 1995), the microwave (COBE; Bennett et al. 1996), and the radio (FIRST; Becker, White, \& Helfand 1995). These surveys improved on numerous earlier surveys in terms of angular resolution and limiting brightness.

Even in energy bands where most of the sky has been mapped, little of the sky is regularly monitored. To date, large portions of the sky are continuously monitored only in

the radio and gamma ray bands. In the radio band, sky monitoring is crudely done, but not stored, by anyone who owns a common radio or (non-cable) television. One more sensitive 
radio sky-monitoring project that is being planned is Argus (Dixon 1993).

In the gamma-ray band, sky monitoring began in the 1960s with the launch of the Vela satellites. A changing armada of Solar System satellites has kept continuous watch ever since. One example of a recent, relatively sensitive all- sky gamma-ray monitor is BATSE onboard the Compton Gamma Ray Observatory. BATSE's 8 detectors monitor the sky visible from low Earth orbit in 16 energy bands stretching from about $25 \mathrm{keV}$ to about $1 \mathrm{MeV}$, with angular resolution ranging from 1.5 to 30 degrees, and with time resolution ranging from 2 microseconds to 2.048 seconds (Fishman et al. 1992). Most of this information is stored and publicly available.

In visible light, sky monitoring has been piecemeal. Paczynski (1996) has discussed scientific attributes of optical sky monitoring projects doing massive photometry. Small regions of the sky are frequently monitored to study stellar and binary star variability. Stellar and binary star photometry began this way last century. Many of these observations are "epochal" in the sense that a given section of the sky is returned to only after a given epoch of time has elapsed. More recently, fields on the order of degrees have undergone epochal monitoring on a daily time scale down to a visual magnitude of about 20 (Udalski et al. 1992, Alcock et al. 1993). These fields have included the LMC and the Galactic Bulge through Baade's window, with tens of millions of stars being monitored allowing hundreds of candidate microlensing events to be recorded.

One survey monitoring part of the sky is the All Sky Automated Survey (ASAS; Pojmanski 1997, Pojmanski 1998), which monitors 140 square degrees a few times a night. Stardial (McCullough \& Thakkar 1997) scans a strip of sky automatically and places images on the internet in near-real time. The Amateur Sky Survey (TASS; Richmond 1997; Richmond et al. 1998) has been scanning the celestial equator with CCD cameras since 1996. Perhaps, though, the most ambitious current epochal sky monitor operating at visible 
wavelengths is the Livermore Optical Transient Imaging System (LOTIS; Park et al. 1997;

Williams et al. 1997). LOTIS currently captures images of roughly $1 / 4$ of the sky, detecting stars as dim as 13th magnitude on a daily basis. An upgraded system dubbed Super-LOTIS has recently begun monitoring part of the optical sky every 21 days, detecting stars as dim as visual magnitude 19. Similarly, the Robotic Optical Transient Search Experiment (ROTSE, Marshall 1997) has planned capabilities to image perhaps $1 / 4$ of the sky to 14 th magnitude on a daily basis.

In this paper, the resources necessary to produce epochal and continuous surveys of the entire sky in are estimated as a function of limiting brightness. Section 2 will discuss the general theoretical concepts that define sky survey limits, and show how they apply to a visible light survey. In $\S 3$ example implementations are discussed for canonical epochal and continuous survey characteristics. In $\S 4$, we describe a single-telescope prototype system that we are using to monitor about 1600 square degrees to visual magnitude 13 . In $\S 5$, scientific discussion and conclusions will be given.

\section{Theory of Sky Monitoring}

\subsection{Constraints}

Realistic continuous sky surveys should have limits defined by the type of scientific return desired. Nevertheless, sky monitoring is subject to practical constraints related to the telescope, the detector, data transfer and storage, the observing site, and the criteria used for detection. General constraints on digital optical sky surveys are discussed, for example, by Kron (1995).

Telescopes are constrained to have a fixed focal length $f$ and a fixed aperture radius $r$ (and hence a fixed focal ratio). Additionally there might be a financial limit on the number 
of identical telescopes $N_{t e l}$ that can be deployed.

Detector constraints include a given pixel pitch $p$, bit depth $\beta$, pixel number $n_{C C D}^{2}$ (for a square CCD array), and quantum efficiency $e_{C C D}$. The CCD and its electronics will give rise to a given dark current noise $D_{c}$, read noise $R_{c}$, and a finite readout rate $R_{\text {readout }}$.

Detection constraints include that at least $\alpha$ pixels exist on the sky for each candidate source, and that a given signal to noise ratio $S / N$ is needed to provide the desired scientific return. Time between observations of the same piece of sky creates the duration of the epoch of observation $t_{\text {epoch }}$.

Storage constraints include a fixed byte size per pixel $b$, a finite amount of data storage available and a limited rate at which data can be taken $R_{\text {data }}$. For example, $R_{\text {data }}$ might be limited by the thinnest data pipe, or by the maximum amount of write-able disk space available in one night.

Site constraints include fraction $e_{n i g h t}$ of allocated time that can be used for direct observation of the sky. Inefficiencies include weather, cloudiness, slew time and the time needed to obtain dark frames.

It will be shown in the subsections below that once a desired limiting brightness is determined, the above constraints will combine to define appropriate observing system parameters ranging from the telescope focal length and the data acquisition rate to the recurrence epochs for epochal and continuous sky surveys.

\subsection{Brightness Limits Survey Pixel Size}

When the number of sources exceeds the number of pixels, the ability to associate specific sources with specific flux changes becomes more difficult. A sky survey could 
proceed even beyond this limit, and even carry significant scientific value, but here we will consider this a practical limit. To insure that dim pixels usually surround bright pixels, the number of pixels should be a large factor $\alpha$ greater than the number of sources. An example value for $\alpha=25$.

The number of pixels $N_{\text {pixel }}$ that tile the whole sky is a function of pixel size. In general, $N_{\text {pixel }}=\Omega_{\text {sky }} / \Omega_{\text {pixel }}$. Given that $N_{\text {pixel }}=\alpha N_{\text {source }}$ at the limiting magnitude, then $\alpha N_{\text {source }}=4 \pi / \Omega_{\text {pixel }}$. Given square pixels and that pixel diameter $\theta_{\text {pixel }}=\sqrt{\Omega_{\text {pixel }}}$ then

$$
\theta_{\text {pixel }} \sim \sqrt{\frac{\pi}{\alpha N_{\text {source }}} .}
$$

For strongly anisotropic source distributions, $\alpha$ will change with sky location.

At the start of the design phase of a sky-monitoring program at any wavelength, the observer's scientific goals might yield a minimum brightness level that is desirable. At this brightness level, the sky surface density of objects at that wavelength should be well known, so that the number of pixels on the sky needed could be determined

In this paper we will consider a canonical example sky survey of monitoring stars in visible light down to a desired limiting magnitude. For light in the visible Johnson $V$ band, Figure 1 shows the expected surface density of stars on the sky for a standard Bahcall-Soneira model of our Galaxy (Bahcall \& Soneira 1980; Bahcall 1986), as a function of limiting visual magnitude. Four lines depict stellar surface densities at the labeled galactic latitudes. Note that densities near the Galactic plane can exceed those near the Galactic pole by more than an order of magnitude.

Figure 1 gives a starting point for the design of a sky monitoring survey. From scientific concerns one chooses a limiting visual magnitude $m_{V}$. Given a field at Galactic latitude $b$, one can find the sky surface density of stars, which can be multiplied by $\alpha$ to give pixel density. Alternatively, to give an all-sky survey uniformity, one might use the average value 
of $\alpha$ for the whole sky.

\subsection{Pixel Size Limits Telescope Pointings}

If the observer chooses a limiting magnitude $m_{V}$ for a survey and plans to use a CCD with a given number of pixels, $n_{C C D}^{2}$, then the minimum number of separate pointings, $N_{\text {point }}$, each of $N_{\text {tel }}$ telescopes must make to image the entire sky can be determined. From last section, the number of pixels needed over the entire sky was determined solely from $m_{V}$ to be $N_{\text {pixel }}$. Now all the pointings of all the telescopes should cover the whole sky so that $N_{\text {pixel }} \leq N_{\text {tel }} N_{\text {point }} n_{C C D}^{2}$. Solving for the number of pointings gives

$$
N_{\text {point }} \geq \frac{N_{\text {pixel }}}{n_{C C D}^{2} N_{\text {tel }}}
$$

A single telescope cannot see more than half the sky from the surface of the Earth. This limits $N_{\text {tel }} \geq 2$, and $N_{\text {point }} \geq 1$.

For a canonical survey in visible light, Figure 2 plots the minimum number of pointings needed to cover the entire sky, as a function of the survey limiting magnitude $m_{V}$. Four telescopes are assumed to be operating simultaneously. The number of sky pixels is chosen by setting $\alpha=25$ pixels per star on the sky down to $m_{V}$. Lines on the plot depict CCD arrays with $n_{C C D}$ of 1024,4096 , and 16,384. Throughout this paper we assume $p$ is a constant for all $n_{C C D}$. The flat part of each curve indicates that a single pointing by each of the four telescopes would create more than 25 pixels per star.

Alternatively, for a continuous survey, the number of pointings is fixed at $N_{\text {point }}=1$, and the above equation can be solved for the minimum number of telescopes needed to view the entire sky simultaneously. $N_{t e l}$ can be discerned from Figure 2 by multiplying $N_{\text {point }}$ by 4: $4 N_{\text {point }}$ then corresponds to the minimum number of dedicated telescopes needed to ensure that the number of deployed detector pixels $\left(N_{t e l} n_{C C D}^{2}\right)$ is greater than the number 
of sky pixels $\left(\alpha N_{\text {star }}\right)$.

\subsection{Pixel Size Limits Data Volume}

The ability to store data could limit the practical extent of a sky monitoring project. Data volume is therefore estimated for epochal and continuous sky surveys. To start, the minimum data obtainable per recurrence epoch is the number of sources at the survey limiting brightness multiplied by the number of bytes used to record each source: $b N_{\text {source }}$. For our canonical visual survey, the dashed line in Figure 3 shows this minimum limit.

The data volume can be written directly in terms of the number of elements in CCD array and the number of observations needed. More specifically,

$$
\text { Data }=b N_{\text {tel }} N_{\text {point }} n_{C C D}^{2} .
$$

In our canonical epochal survey in visible light, $N_{t e l}=4$ and $\alpha=25$. For these assumptions, a plot of data volume per sky scan versus $m_{V}$ is given in Figure 3 for our three canonical square CCD arrays. The flat part of each curve indicates magnitudes where a single pointing of all four survey telescopes would create the needed number of sky pixels.

Figure 3 indicates that CCD arrays with the smallest number of pixels create the least data for sky scans at bright limiting magnitudes. Sky scans sensitive only to stars brighter than magnitude 15 would create only several Gigabytes of data, which might be conveniently stored in present day 8-mm tapes.

At faint magnitudes, the number of pixels in the CCD array is irrelevant. It is, however, much more difficult to store these scans on a single storage medium with technology easily available in 1999. Perhaps when data storage devices increase by a factor of about 100 , easy data storage of sky scans to magnitude 20 may be possible. Were data storage limits 
to increase by a factor of 1.7 each year (Moore's Law), it would then be convenient to store such information in roughly 9 years, in the year 2008.

\subsection{Pixel Size Limits Telescope Focal Length, Aperture}

The angular size of a pixel on the sky combines with the physical size of a pixel in the detector to define a unique telescope focal length. Given that each pixel of the CCD array has pitch $p$, then the focal length of each telescope must be

$$
f=\frac{p}{\sqrt{\Omega_{\text {pixel }}}} .
$$

Since $f$ is directly proportional to $p$, it is straightforward to extrapolate this plot to larger values of $p$. If we further demand that each telescope has focal ratio $F=f /(2 r)$, we see that the aperture of each telescope must be $r=f /(2 F)$. Again, since $r$ is directly proportional to $f$, it is straightforward to extrapolate $r$ for different values of $F$.

For our canonical visible-light surveys, a plot of $f$ versus $m_{V}$ for different values of $n_{C C D}$ is given in Figure 4. As with previous plots, the flat part of each curve indicates magnitudes where a single pointing of all four epochal survey telescopes would create the needed number of sky pixels. At faint magnitudes, as $m_{V}$ approaches magnitude 20, the focal length of the telescopes needed approach one meter. At bright magnitudes, the focal length can be a centimeter or less - much smaller than common with conventional telescopes. We note that camera with such focal lengths are neither impossible nor unprecedented in astronomy. Cameras like this are popularly referred to as "wide-angle" or "fish-eye." Such a camera would have similarities to the human eye.

Every telescope's angular resolution is limited by diffraction. A convenient parameter quantifying an angle where diffraction effects become important is $\theta_{\text {diffraction }}=1.22 \lambda /(2 r)$, where $\lambda$ is the average wavelength of light being observed. For an $f / 2$ telescope and light 
with wavelength of $5 \times 10^{-7}$ meters, the area in Figure 4 limited by diffraction would be below the dashed line. Figure 4 demonstrates that pixel sizes are so large in the optical surveys described that diffraction limits are not generally important: stars are assumed to be imaged inside a single pixel with a suitably sophisticated optical system design. Furthermore, blurring by the Earth's atmosphere, which would obviate the use of any pixels less than on order $\theta_{\text {pixel;min }}=2$ arcseconds, will not be important in any example surveys discussed.

\subsection{Brightness Limits Integration Time}

In a survey in any wavelength band, the objects being surveyed must be viewed for a finite time to build up a signal necessary to analyze them. This amount of time is a strong function of the telescope and detector being deployed. The case of stars in visible light is analyzed in some detail below.

The apparent luminosity of a star of magnitude $m$ can be written $I_{*}=I_{\odot} 10^{\left(m_{\odot}-m\right) / 2.5}$ counts $\mathrm{cm}^{-2} \mathrm{sec}^{-1}$ where the $*$ subscript refers to the star and the $\odot$ subscript refers to the Sun. For visible light (Johnson $V$ ), the relation between visual magnitude and flux detectable over the visual band pass at the top of the Earth's atmosphere can be found from relations in Zombeck (1990) to be $I_{*, s p a c e}=8.5 \times 10^{5} 10^{-m_{V} / 2.5}$ counts $\mathrm{cm}^{-2} \mathrm{sec}^{-1}$. From the Earth's surface, a star visible in a clear sky at zenith will cross one air mass and appear $\Delta m_{V}=0.2$ magnitudes more faint (Allen 1976). Since we are interested in stars at various zenith angles, we will consider that a canonical star crosses two air masses and hence appears 0.4 visual magnitudes more faint:

$$
I_{*}=\left(5.9 \times 10^{5} \text { counts } \mathrm{cm}^{-2} \mathrm{sec}^{-1}\right) 10^{-m_{V} / 2.5} .
$$


The total counts received from this star are

$$
C_{*}=4 r^{2} t e_{C C D} I_{*}=1.2 \times 10^{6}\left(\frac{r}{1 \mathrm{~cm}}\right)^{2}\left(\frac{t}{1 \mathrm{sec}}\right)\left(\frac{e_{C C D}}{0.5}\right) 10^{-m_{V} / 2.5}
$$

where $t$ is the duration of the observation, and $e_{C C D}$ is the efficiency of the CCD array. The factor of four is included because it is assumed that the CCD array is square, not round.

The brightness of the background sky varies greatly with respect to time and location. Given a sky brightness of $m_{\text {sky }}$ in magnitudes $\operatorname{arcsec}^{-2}$, the apparent luminosity in the Johnson $\mathrm{V}$ band pass is given by

$$
I_{s k y}=\left(8.5 \times 10^{5} \text { counts } \mathrm{cm}^{-2} \mathrm{sec}^{-1} \operatorname{arcsec}^{-2}\right) 10^{-m_{s k y} / 2.5}
$$

Note that atmospheric effects that degrade star brightness are excluded here.

Now the telescope field of view is

$$
\Omega=(4 \pi \text { steradians }) F_{s k y}=\left(5.3 \times 10^{11} \operatorname{arcsec}^{2}\right) F_{s k y}
$$

where $F_{s k y}$ is the fraction of the sky visible to the telescope. The field of view visible to a single pixel of width $p$ in a telescope of focal length $f$ is $\Omega_{\text {pixel }}=\arctan (p / f) \sim p / f$, assuming the field of view is small compared to a radian. Given a square pixel array of $n_{C C D}^{2}$ pixels, the total field of view of the telescope would be

$$
\Omega=\frac{n_{C C D}^{2} p^{2}}{f^{2}} \sim(1.05 \text { steradians })\left(\frac{n_{C C D}}{1024}\right)^{2}\left(\frac{p}{10^{-5} \mathrm{~m}}\right)^{2}\left(\frac{1 \mathrm{~cm}}{f}\right)^{2}
$$

and the fraction of sky visible would be $F_{s k y}=\Omega /(4 \pi)$.

The total counts received from the sky are then

$$
C_{s k y}=4 r^{2} t \Omega e_{C C D} I_{s k y}
$$

Written in more familiar terms

$$
C_{s k y}=1.7 \times 10^{6}\left(\frac{r}{1 \mathrm{~cm}}\right)^{2}\left(\frac{t}{1 \mathrm{sec}}\right)\left(\frac{\Omega}{1 \operatorname{arcsec}^{2}}\right)\left(\frac{e_{C C D}}{0.5}\right) 10^{-m_{s k y} / 2.5} .
$$


For most observations, the signal is $S=C_{*}$. The noise, however, must include contributions not only from the background sky counts but also counts created during readout and dark time, as well as photon noise. Dark current in the CCD will produce a linearly increasing amount of counts with time: $C_{d a r k}=D t$, where $D$ might be proportional to the ambient temperature. In sum, the noise is given by

$$
N=\sqrt{C_{*}+C_{s k y}+C_{r e a d}+C_{d a r k}}
$$

Scientific observations of a star are usually done above a given signal to noise ratio $(S / N)$. Source detection might be accomplished at $S / N=3$, while photometry might

demand $S / N=100$. The above equations will therefore be solved for the time $t$ needed to create a given $S / N$ for a star of magnitude $m$. This time will be referred to as $t_{m}$.

\subsection{CCD Saturation Limits Exposure Number}

Detectors cannot integrate for an arbitrary long period, as they will eventually saturate. Detector saturation sensitivity can be characterized by the number of bits with which it can record intensity: bit depth $\beta$. The number of corresponding intensity levels is $2^{\beta}$. As signal is related to noise level by the minimum signal to noise ratio that the observer will allow: $S / N$, it follows that $S+N=2^{\beta}$ counts.

For our canonical survey, we will assume a commonly available detector with a depth of 16 bits. We will also assume that the scientific goals of our canonical survey demands a $S / N=100$. These attributes combine to limit the maximum number of signal counts per single detector integration period to about $S \sim 21,400$. The dynamic range in signals detectable above $S / N=100$ is therefore about 214, which corresponds to a span of about 5.8 magnitudes.

To reach the limiting magnitude of a survey, multiple exposures might be needed. 
For our canonical survey, we will assume that each exposure lasts until a source at the limiting brightness nearly saturates a CCD pixel. Alternatively, some surveys might want to underexpose some frames in an effort to accurately measure the luminosity of bright sources.

After each exposure, the total signal and total background are recalculated, and a new $S / N$ is computed for a source at the survey limiting brightness. After $N_{\text {exposure }}$ exposures, the desired $S / N$ is reached, and a telescope in an epochal survey may be re-pointed. We note that a telescope in a continuous survey will just continue on to new exposures of the same part of the sky, building up sensitivity to increasingly dim objects. In general, the dimmer the source, the lower the temporal resolution. Nevertheless, the continuous survey is somewhat optimized for detection of sources brighter than the survey limiting magnitude because of source confusion.

The integration time $t_{m}=t_{\text {integration }}$ needed to observe a portion of the sky down to the labeled limiting $\mathrm{V}$ magnitude $m$ is shown in Figure 5. Telescope parameters at each limiting magnitude were defined by that magnitude as discussed above for our canonical visual survey. A detector efficiency of $e_{C C D}=0.5$ was also assumed, and detector read noise $C_{\text {read }}$ was taken to be negligible. Note that this integration time is the same for both the described epochal and continuous survey.

\subsection{Efficiencies Limit Survey Recurrence Times}

The timing and duration of sky surveys is limited not only by integration time $t_{m}$, but also by "inefficiencies" that are inherent in realistic observations. Such inefficiencies include the time needed to take a dark frame after each sky exposure $t_{d a r k}$, the time needed to readout the CCD array after each exposure $t_{\text {readout }}$, and the time spent waiting 
for a clear and dark sky, here parameterized as $e_{\text {night }}$. Now for a single exposure,

$t_{\text {readout }}=b n_{C C D}^{2} / R_{\text {readout }}$, where $R_{\text {readout }}$ is the readout rate of the CCD in bytes $\sec ^{-1}$. The total time needed to complete one set of observations to the survey limit $m$ would then be

$$
t_{1}=N_{\text {exposure }}\left(t_{m}+t_{\text {dark }}+2 t_{\text {readout }}\right) \text {. }
$$

For an epochal survey, the total time between observations of the entire sky will be $t_{\text {epoch }}=e_{\text {night }} N_{\text {point }} t_{1}$, where $e_{\text {night }}$ is an observing efficiency: the fraction of time during which observations can actually occur. Combining above equations

$$
t_{\text {epoch }}=\frac{N_{\text {point }} t_{1}}{e_{\text {night }}} .
$$

For our canonical optical survey with the added constraint that $e_{\text {night }}=0.25$, Figure 6 plots $t_{\text {epoch }}$ verses $m_{V}$. Note that the "turnover" point where $n_{C C D}=16,384$ is favored over $n_{C C D}=4096$ occurs at approximately magnitude 13 for an epochal survey.

For a continuous sky survey, $t_{\text {continuous }}$ is the time it takes to complete one set of sky exposures together sensitive to magnitude $m_{V}$ at a given $S / N$. This differs from $t_{\text {epoch }}$ since more telescopes are deployed, relaxing the demand on any one telescope to a single pointing. In other words,

$$
t_{\text {continuous }}=\frac{t_{1}}{e_{\text {night }}}
$$

A plot of $t_{\text {continuous }}$ versus $m_{V}$ is given in Figure 7 for our canonical optical survey.

\subsection{Data Volume and Survey Times Limits Data Rate}

The data volume generated by a single scan of the sky (as depicted in Figure 4 for our canonical survey) is possibly of less interest than the data accumulation rate. The data rate is simply the data volume recorded over the recurrence time for the survey. For an epochal 
survey,

$$
R_{\text {epoch }}=\frac{\text { Data }}{t_{\text {epoch }}}
$$

Figure 8 plots a minimum $R_{\text {epoch }}$ versus $m_{V}$ for our canonical survey. Inspection of this plot indicates that $R_{\text {epoch }}$ actually decreases as $m_{V}$ dims. This is true so long as data from intermediate exposures is not saved.

Inspection of Figure 8 shows that $R_{\text {epoch }}$ is at first constant at bright magnitudes. The curve flatness is created by the need for only a single exposure and pointing to reach the desired limiting magnitude. The decrease in $R_{\text {epoch }}$ at slightly fainter magnitudes is caused by the time needed for repeated exposures, although still only a single pointing from each of the four telescopes is required. Finally, near magnitude 20, both multiple pointings to different parts of the sky and multiple exposures of each part of the sky are required. That $R_{\text {epoch }}$ decreases at fainter magnitudes results from the net observation time increasing at a greater rate than the data volume.

It appears that the data rate created by any of the considered epochal surveys is not enough to challenge even present day data storage media. The Gigabyte storage capacity of modern day 8-mm tapes could reasonably absorb the data generated.

The data rate for a continuous sky survey would be

$$
R_{\text {continuous }}=\frac{\text { Data }}{t_{\text {continuous }}}
$$

A plot of $R_{\text {continuous }}$ versus visual limiting magnitude for our canonical visual survey is given in Figure 9. Note that the data rate is higher than in the epochal case, since there are now more than four telescopes accumulating data simultaneously. The combined increase in both data volume and $N_{\text {tel }}$ will cause $R_{\text {continuous }}$ to increase at the very faintest limiting magnitudes considered. At this faint end, the extra telescopes needed using smaller pixel arrays would actually take more time to complete their work than fewer telescopes mounted 
with a larger pixel arrays. Still, for all but the smallest pixel array at the faintest limiting magnitude considered, data storage on the Gigabyte level per night would be needed, well within the storage capacity of modern 8-mm tapes.

\section{Canonical Implementations}

\subsection{An Example Epochal Sky Survey}

Attributes of a specific epochal and continuous all-sky survey down to visual limiting magnitude of $m_{v}=15$ will now be computed as an example of the above results. We will start by assuming telescope and CCD parameters common in 1999. Defining attributes will therefore include a CCD array that has $n=4096^{2}$ pixels each with pitch $p=10^{-5}$ meters.

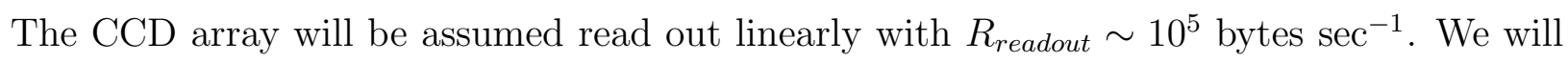
demand $\alpha=25$ pixels per star for star on the sky brighter than the magnitude limit. Four survey $f / 2$ telescopes, operating in parallel, will be assumed.

The magnitude limit of our survey will dictate the minimum pixel density we will use on the sky. For simplicity, we will use a constant pixel density over the sky, although it is possible to define surveys where pixel density changes to match star density. Alternatively, it is also possible to use the pixel density to determine the limiting survey magnitude, for example integrating longer in areas of lower stellar densities.

At magnitude 15, we note that Figure 3 indicates there are about 20 million stars on the sky brighter than $m_{V}=15$. Given $\alpha=25$ pixels per star, we might then expect about 500 million pixels to be needed on the sky. A more precise estimate yields $N_{\text {pixel }}=5.17 \mathrm{x}$ $10^{8}$ pixels will be needed.

Now each of our 4 telescopes has $4096^{2}$ pixels, yielding $6.7 \times 10^{7}$ pixels in total. Therefore, at least from the information standpoint, each telescope must re-point at least 8 
times to record the entire sky. Assuming each pixel generates 4 bytes of data, each sky scan should generate on order 2.1 Gigabytes. This information is discernable from Figure 3.

Given the above pixel pitch of $p=10^{-5} \mathrm{~m}$, Equation 4 allows us to compute the focal length of the (assumed identical) telescopes. Each sky pixel would take up about $\Omega_{\text {pixel }} \sim 4 \pi / N_{\text {pixel }} \sim 2.4 \times 10^{-8}$ steradians, so that $f \sim p / \sqrt{\Omega_{\text {pixel }}} \sim 0.064$ meters. Given all telescopes are $f / 2$, the diameter of each telescope would also be 0.032 meters. This is also evident in Figure 4.

We will (again) assume that the CCD is 16 bits deep, and has detection efficiency of $e_{C C D}=0.5$. We will assume that the background sky glows at 21 magnitudes $\operatorname{arcsec}^{-2}$. The exposure time needed to reach visual magnitude $m_{V}=15$ at a signal to noise $S / N=100$ is $t_{\text {integration }}=20,598$ seconds (about 5.7 hours), more than the 2974 seconds (about 49.6 minutes) needed to saturate the CCD. Therefore at least seven separate exposures will be needed.

Integration time is not necessarily the whole story, however. The time to take dark frames, readout times, and the general efficiency of operation would combine to determine the true recurrence epoch of this all-sky survey. Given a $R_{\text {readout }}=1.0 \times 10^{5}$ bytes per second, a single exposure with a single telescope would take $t_{\text {readout }} \sim 671$ seconds to readout. Assuming that a dark frame was required to be taken every time, with the same integration and readout times, the total time for one saturated exposure would be about 7290 seconds, or about 2 hours. Given 6 near-saturated exposures per pointing and one additional exposure calculated to acquire the survey magnitude limit, a complete scan of the sky could be completed in 14 hours. Given slightly more optimistic limits, a single complete set of sky exposures photometrically sensitive to $m_{V}=15$ could be achieved in a single night. Including a general time efficiency (including daytime and cloudiness) of $e_{\text {night }} \sim 0.25$, however, the total time for a single pointing is $t_{1}=50,593$ seconds. Given 8 
pointings per telescope needed to cover the entire sky, the recurrence epoch of this survey would run about $t_{e p o c h} \sim 1.6 \times 10^{6}$ seconds, or under 19 days. This can be seen in Figure 6 .

\subsection{An Example Continuous Sky Survey}

Let's now assume that we want to create a continuous record of the entire sky. Continuous monitoring would accumulate data so long as daylight, dark time, and clouds will allow. (Even daylight records might be of interest to meteorologists.) Each telescope will then be dedicated to a fixed area of the sky. Such dedication might lower systematic errors by reducing pointing errors, simplifying data analysis, and reducing telescope complexity.

An example continuous sky survey sensitive to $m_{V}=15$ at $S / N=100$ would be quite similar to the epochal survey discussed above. A major difference would be the number of telescopes deployed. In the epochal survey, 4 telescopes were re-pointed 8 times each. In the corresponding continuous survey, given the same $n_{C C D}$, we would need 32 separate telescopes. Possibly, the cost of mass producing and deploying 32 identical telescopes might be less than 32 times the cost of one survey telescope. Alternatively, a continuous survey might undergo incremental implementations, and so not cover the entire sky initially.

The number of pixels generated per sky scan will be the same as with the epochal survey. Given $b=4$ bytes per pixel, the data generated would still be $b N_{\text {pixel }} \sim 2.1$ Gigabytes. The focal length of each $f / 2$ telescope would again be $f=0.064$ meters, and the radius $r=0.016$ meters.

As above, we will also assume that the CCD is 16 bits deep and has detection efficiency of $e_{C C D}=0.5$, and assume that the sky background is 21 magnitudes $\operatorname{arcsec}^{-2}$. The main difference between our example epochal and continuous surveys comes in the recurrence 
time. The extra-dedicated telescopes of the continuous survey will cut the time down between re-imaging identical patches of the sky. Since each telescope need do only one pointing instead of 8 , the recurrence time is reduced by a factor of 8 . The recurrence time is therefore reduced from about 19 days, to about 2.3 days. Increases in temporal frequency would require additional telescopes of larger aperture, hence, for constant f/ratio, smaller fields of view.

\subsection{The Potential of Future Sky Monitors}

What technology development is needed to motivate the creation of a continuous record of the entire sky? This is strongly a function of the wavelength band in question. In general, the development of CCDs and the technological replacements for CCDs should be a leading factor.

One potential limiting factor is the readout rate of CCD arrays. Figure 6 indicates that at a given limiting magnitude, it is not efficient to use a large CCD array, due predominantly to the time it takes to readout the large amount of data. Although physical processes fundamentally limit this time, recent progress has been made in reading out CCD sections in parallel. Were readout times reduced significantly or even eliminated, integration time $t_{m}$ would become more closely related to the recurrence time of a sky scan.

Possibly most relevant to the future of astronomical sky surveys appears is the growth in size of detector arrays. Gains in efficiency of CCDs, already 50\% to $80 \%$, or decreasing pixel pitch $p$, (which would decrease the photon-capture cross-sectional area, requiring longer exposure times to reach the same limiting magnitudes), or other chip related technology offer only marginal advances. Larger pixels than those commonly available (roughly $30 \mu \mathrm{m}$ ), might be useful, however, particularly at brighter magnitudes. 
As CCD pixel number increases it becomes possible to record an increasing number of stars with a single telescopic system. Theoretically, an old CCD array could just be swapped out for a newer more densely packed array, assuming that the optical system were designed with this eventuality in mind. This would allow a survey telescope to go deeper, although both integration time and recurrence epoch would necessarily increase.

A simple and perhaps compelling method of predicting the limiting magnitude of sky surveys is to assume pixel number as the single greatest limiting feature. When pixel number becomes on the order of the number of stars at a given magnitude, then a photometric measurement of those stars will be assumed feasible in a single night. Inherent in this assumption is that readout rates will stay unimportant as it becomes cheaper to add additional parallel output pipes. Lastly, we assume that data storage tapes and drives increase sufficiently to allow inexpensive long-term data storage.

McCall \& Corder (1995) have discussed how CCD array technology has increased over the past 30 years, even though CCDs did not exist in present form 30 years ago. We here parameterize this as a factor of 1.7 every year.

In 1999, we will take to be representative of easily obtained arrays $n_{C C D}=4096^{2} \sim 1.7$ x $10^{7}$ elements. In Figure 3, the dashed line depicts the data amount expected were each star to need $b=4$ bytes of data storage. Dividing this byte size by $b$, we can see the base number of stars in the Bahcall-Soneira model of our Galaxy. The above pixel element amount therefore corresponds to about $10^{7}$ stars, which occurs at a limiting visual magnitude of $m_{V} \sim 12$. Given the above pixel factor rate increase, we expect that a survey to 15th magnitude in a single night will be possible with common resources in between two and three years, and to 20th magnitude in a single night will be possible in about 12 years, by about 2011 . 


\section{A Prototype System}

To explore both the feasibility of obtaining a continuous record of the entire night sky, as well as to familiarize ourselves with realistic operational constraints and data handling issues, we have constructed CONtinuous CAMera (CONCAM) 1, shown in Figure 10. CONCAM 1 was built from readily available components for a cost of under $\$ 5 \mathrm{~K}$. It is composed of a 8-mm f/4 fisheye lens, a Meade 8-inch LS-200 mount, and an ST-7 with 765 x 510 pixels each with pitch 6.9 microns x 4.6 microns. A laptop computer running CCDOPS operates CONCAM 1. The resulting field of view is 32 degrees $\mathrm{x} 49$ degrees.

CONCAM 1 tracks the sky at the sidereal rate. Exposures are obtained continuously, allowing for readout intervals. Data is stored first on the laptop hard drive but later transferred to read/writable CD-ROMs. Flat field exposures and dark frames are obtained regularly. A typical exposure from CONCAM 1 is shown in Figure 11. Much of the Ursa Major is visible, and the bright star near the frame's center is Dubhe ( $\alpha \mathrm{UMa})$.

CONCAM 1 can be described in terms of the quantities defined in Section 2. The (geometric) mean number of pixels is $n_{C C D}=\sqrt{765 \times 510} \sim 624$. Similarly, the mean pixel pitch $p \sim 5.6 \mu \mathrm{m}$. The camera sees $\Omega_{t e l}=0.48$ steradians of the sky, giving it a mean angular side length of the field of view of $\theta_{t e l} \sim 39$ degrees. Therefore, at least $N_{t e l}=27$ telescopes would be needed to monitor the complete sky simultaneously. If only $N_{t e l}=4$ telescopes were deployed, each telescope would need to be repointed a minimum of $N_{\text {point }}=7$ times. These cameras would divide the entire sky into $1.05 \times 10^{7}$ pixels. If we demand $\alpha=25$ pixels per source, $N_{\text {source }}=4.2 \times 10^{5}$ sources can be independently

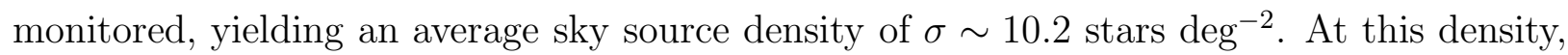
source confusion would begin at about $m_{V} \sim 10$ in directions toward the Galactic plane, at about $m_{V} \sim 15$ in directions toward the Galactic poles.

In the near future, CONCAM 2 will be created from an ST-8 CCD camera, and will 
feature quadruple the static angular coverage of CONCAM 1.

\section{Discussion and Conclusions}

Technological progress is naturally continuing in a direction that is making a continuous record of the entire sky feasible. Perhaps forethought now will help emphasize scientific questions currently at the forefront of current astronomy, and help organize observation attributes and data storage methods useful far into the future.

Continuous sky surveys to bright magnitudes are currently feasible. Projects like LOTIS, ROTSE, and ASAS are already engaging in partial epochal surveys. Technology exists today that would enable an all-sky continuous surveys at bright visual magnitudes. For example, there is no fundamental reason why astronomers cannot keep running a continuous daily survey of the entire sky brighter than magnitude 15, and of millions of stars visible away from the glare of the Sun.

Technology does not usually proceed at a linear (or even logarithmic) pace, as technological inventions and procedural innovations often create leaps in what is possible. Alternatively, progress in a field may slow as physical limits are approached. For example, one potentially relevant technological innovation includes the development of CMOS detection chips.

Possible scientific returns of a recorded continuous and epochal sky monitoring are many and varied, ranging from a better understanding of unusual variable stars to discovery of potential doomsday asteroids (Paczynski 1996). It is probable that not all-scientific returns can be foreseen. Nevertheless, other potential scientific discoveries of a continuous record of the entire sky might include the establishing of case histories for variables of future interest, uncovering new forms of stellar variability, discovering the brightest cases 
of microlensing (Nemiroff 1998), discovering new novae and supernovae, discovering new counterparts to gamma-ray bursts, monitoring known Solar System objects, and discovering new minor-planets.

Limiting brightness was the starting point for sky survey designs discussed in this paper. Other scientifically chosen observables, such as intrinsic source timing or spectral qualities, might also be considered starting points for the design of sky surveys. In many cases the above framework might still prove useful. A computer code from which most of the results in this paper can be reproduced has been submitted to the Astrophysics Source Code Library (ASCL.NET).

We thank C. Ftaclas and for helpful discussions and comments, and W. Pereira for help with instrumental logistics. RJN thanks B. Paczynski for discussions where he found that he and others already had many of the same ideas discussed above. This research was supported by grants from NASA and the NSF. 


\section{REFERENCES}

Alcock, C. et al. 1993, Nature, 365, 621

Allen, C. W. 1976, Astrophysical Quantities (London, UK: Athlone Press)

Antia, B. 1993, J. Interplanetary Soc. 46, 9

Bahcall, J. 1986, ARA\&A, 24, 577

Bahcall, J. N. \& Soneira, R. M. 1980, ApJS, 44, 73

Becker, R. H., White, R. L., \& Helfand, D. J. 1995, ApJ, 450, 559

Bennett, C. L. et al. 1996, ApJ, 464, L1

Dixon, R. S. 1993, 44th IAF/IAC Congress, Austria, 9

Fichtel, C. 1996, Astron. Ap. Supp. 120, 23

Fishman, G. J. et al. 1992, in: Gamma-ray bursts; Proceedings of the Workshop, Univ. of Alabama, Huntsville, Oct. 16-18, 1991 (A93-40051 16-93), p. 13-21

Gaustad, J., Rosing, W., Chen, G., McCullough, P. \& Van Buren, D. 1997, BAAS, 190, \# 30.05

Gunn, J. E. 1995, BAAS, 186, \# 44.05

Kron, R. 1995, PASP, 107, 766

Marshall, S. et al. 1997, BAAS, 191, \# 48.15

McCall, G. H. \& Corder, J. A. 1995, New World Vistas, Air and Space Power for the 21st Century, Sensors Volume, USAF Scientific Advisory Board, 15 Dec 1995

McCullough, P. R. \& Thakkar 1997, PASP, 109, 1264 
Nemiroff, R. J. 1998, ApJ, 509, 39

Paczynski, B. 1996, 12th IAP Colloquium: Variable stars and the astrophysical returns of microlensing surveys, Paris, IAP, July 7-12, 1996, astro-ph/9609073

Park, H.-S. et al. 1997, ApJ, 490, 99

Pojmanski, G. 1997, Acta Astronomica, 47, 467

Pojmanski, G. 1998, Acta Astronomica, 48, 35

Reid, I. N. et al. 1991, PASP, 103, 661

Richmond, M. 1997, BAAS, 190, \# 33.05

Richmond, M. et al. 1998, BAAS, 192, \# 55.08

Skrutskie, M. F. et al. 1995, BAAS, 187, \# 75.07

Udalski, A., Szymanski, M., Kaluzny, J., Kubiak, M., and Mateo, M. 1992, Acta Astronomica, 42, 253

Williams, G. G. et al. 1997, BAAS, 191, \# 126.06

Wood, K. S. et al. 1984, ApJS, 56, 507

Zombeck, M. V. 1990, Handbook of Space Astronomy and Astrophysics, Second Edition (Cambribdge, UK: Cambridge University Press) 
Fig. 1. - The surface density of stars on the sky plotted as a function of limiting visual magnitude. A standard Bahcall-Soneira model for our Galaxy was assumed. The four lines correspond to the labeled Galactic latitude. When the surface density of pixels exceeds the surface density of stars, it becomes difficult to monitor stars individually.

Fig. 2.- The minimum number of telescope pointings needed to image the entire sky as a function of the survey limiting magnitude. Four telescopes and twenty-five pixels per disk star are assumed. The three lines correspond to pointing numbers for telescopes utilizing square CCD arrays containing 1024 pixels on a side, 4096 pixels, and 16,384. Larger pixel arrays can tile the sky with the needed number of pixels in fewer pointings. For a continuous sky survey, $4 N_{\text {point }}$ corresponds to the minimum number of dedicated telescopes needed to ensure that the number of deployed detector pixels $\left(N_{t e l} n_{C C D}^{2}\right)$ is greater than the number of sky pixels $\left(\alpha N_{\text {star }}\right)$.

Fig. 3. - The minimum amount of data storage needed to record one image of the entire sky as a function of survey limiting magnitude. The dashed line represents a storage minimum, assuming 4 bytes per star.

Fig. 4.- For a given angular pixel size on the sky and physical pixel pitch in the detector, a unique telescope focal length is implied. This focal length is plotted against the survey limiting magnitude. The dashed line represents a theoretical diffraction limit.

Fig. 5.- The integration time needed to observe a portion of the sky down to the labeled limiting magnitude. An f/2 telescope with focal length depicted in Figure 4 was combined with a square pixel arrays of 1024, 4096, and 16,384 elements on each side.

Fig. 6. - The recurrence time of an epochal survey used to monitor the entire sky down to the labeled limiting magnitude, using three different CCD arrays. In the epochal survey depicted, 4 telescopes are continually repointed to tile the sky to the desired limiting 
magnitude. The epoch of recurrence includes data readout time, dark frame time, and an estimated efficiency of observation of $25 \%$. Canonical survey assumptions include 25 pixels per disk star, a background sky brightness of 21 magnitudes $\operatorname{arcsec}^{2}$, and a pixel number labeled by each curve. The flat portion of each plot is caused by a single integration being able to reach to survey limiting magnitude.

Fig. 7.- The recurrence time of a continuous survey used to monitor the entire sky down to the labeled limiting visual magnitude, using three different CCD arrays. The same defining telescope and CCD parameters are assumed as used in Figure 7, with the exception that the number of telescopes has been increased so that each telescope need not do more than a single pointing.

Fig. 8. - The average data rate for an epochal survey of limiting visual magnitude $m_{V}$.

Fig. 9.- The average data rate for a continuous survey of limiting visual magnitude $m_{V}$.

Fig. 10.- A picture of CONCAM 1, a prototype camera that monitors the sky. In the LANL preprint version, this figure appears as a seperate gif file named concampic.gif.

Fig. 11.- A picture of the sky taken by CONCAM 1. Dubhe, the brightest star in Ursa Major, is visible near the field center. In the LANL preprint version, this figure appears as a seperate gif file named conskypic.gif. 


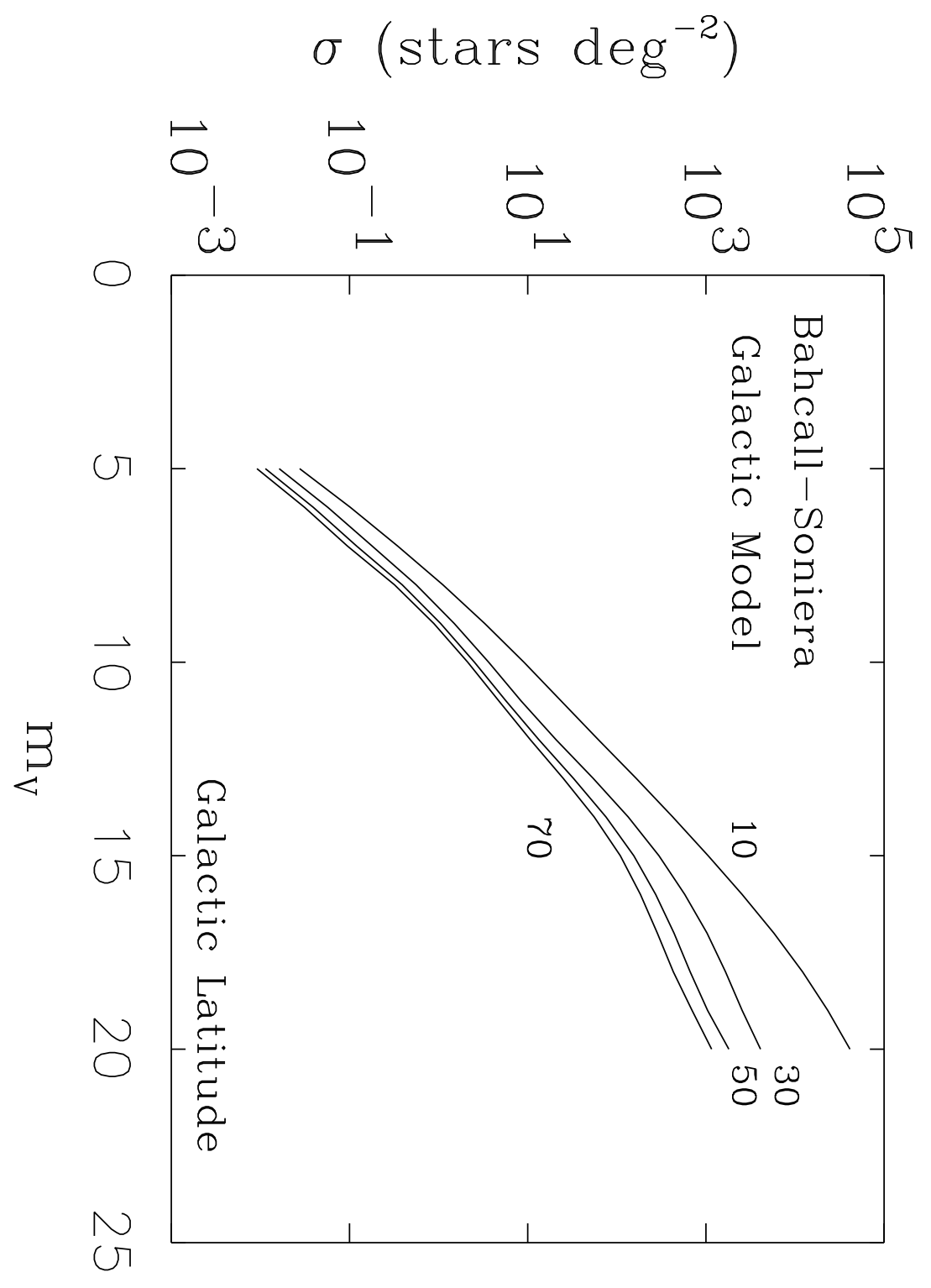




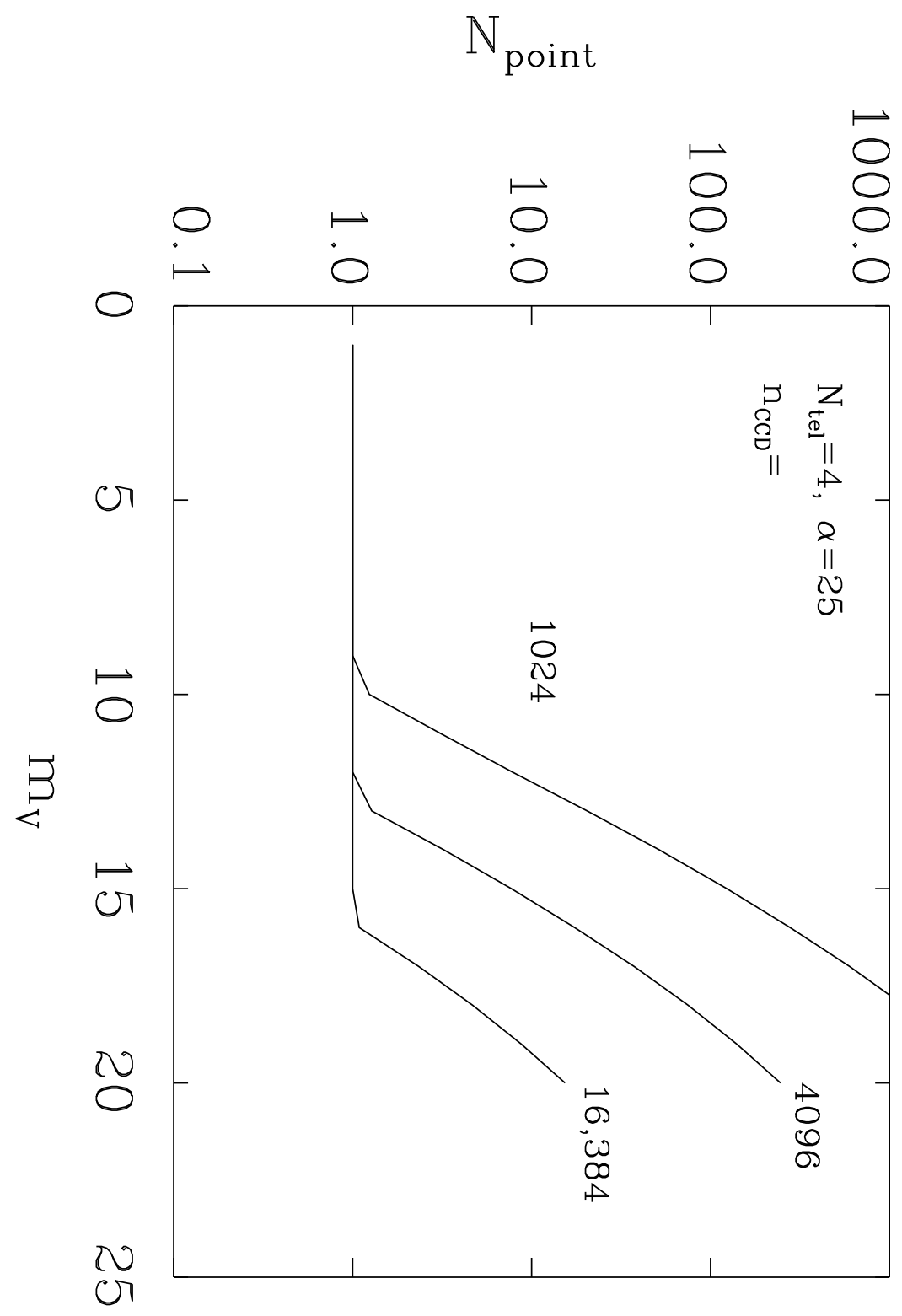




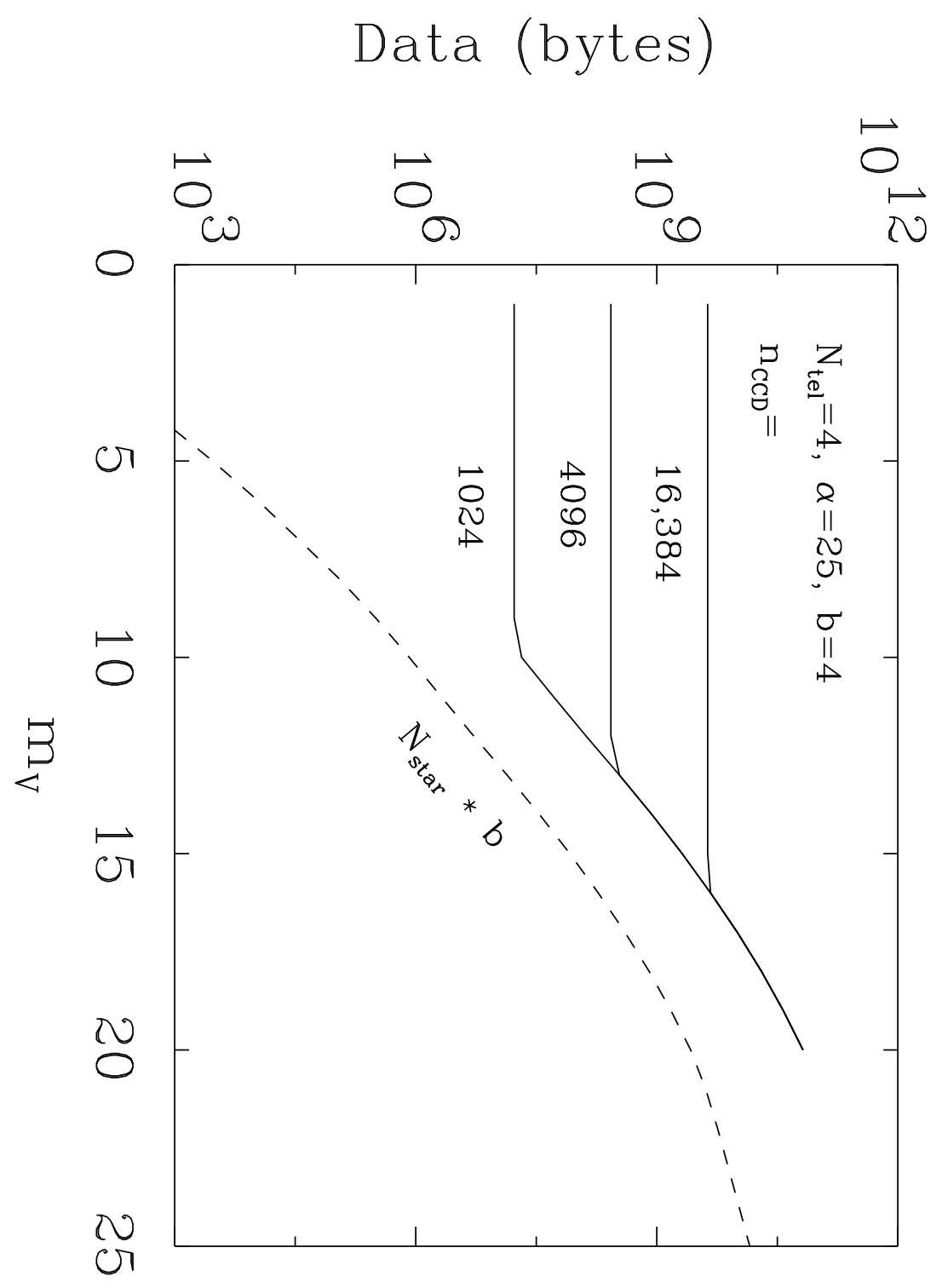




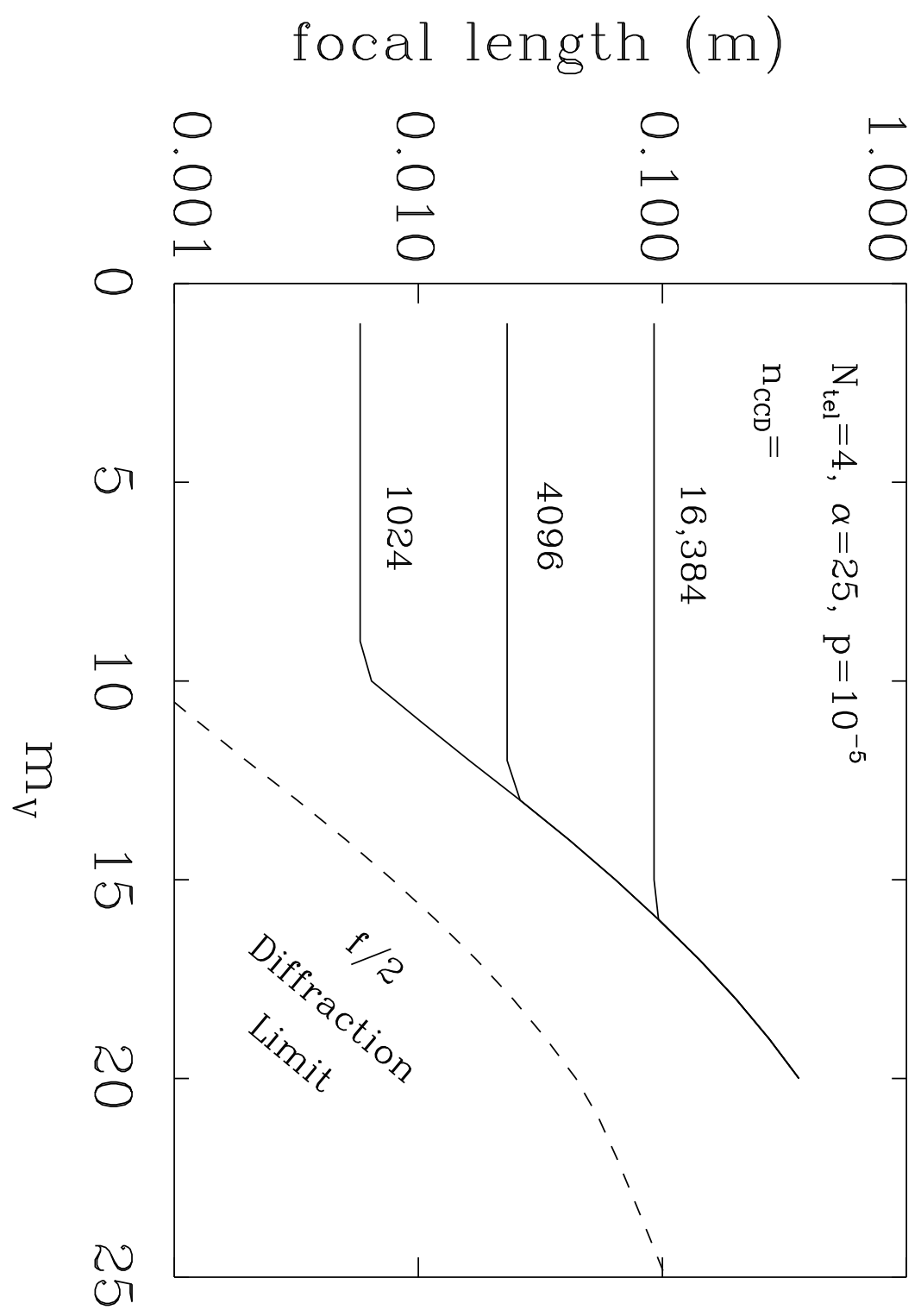




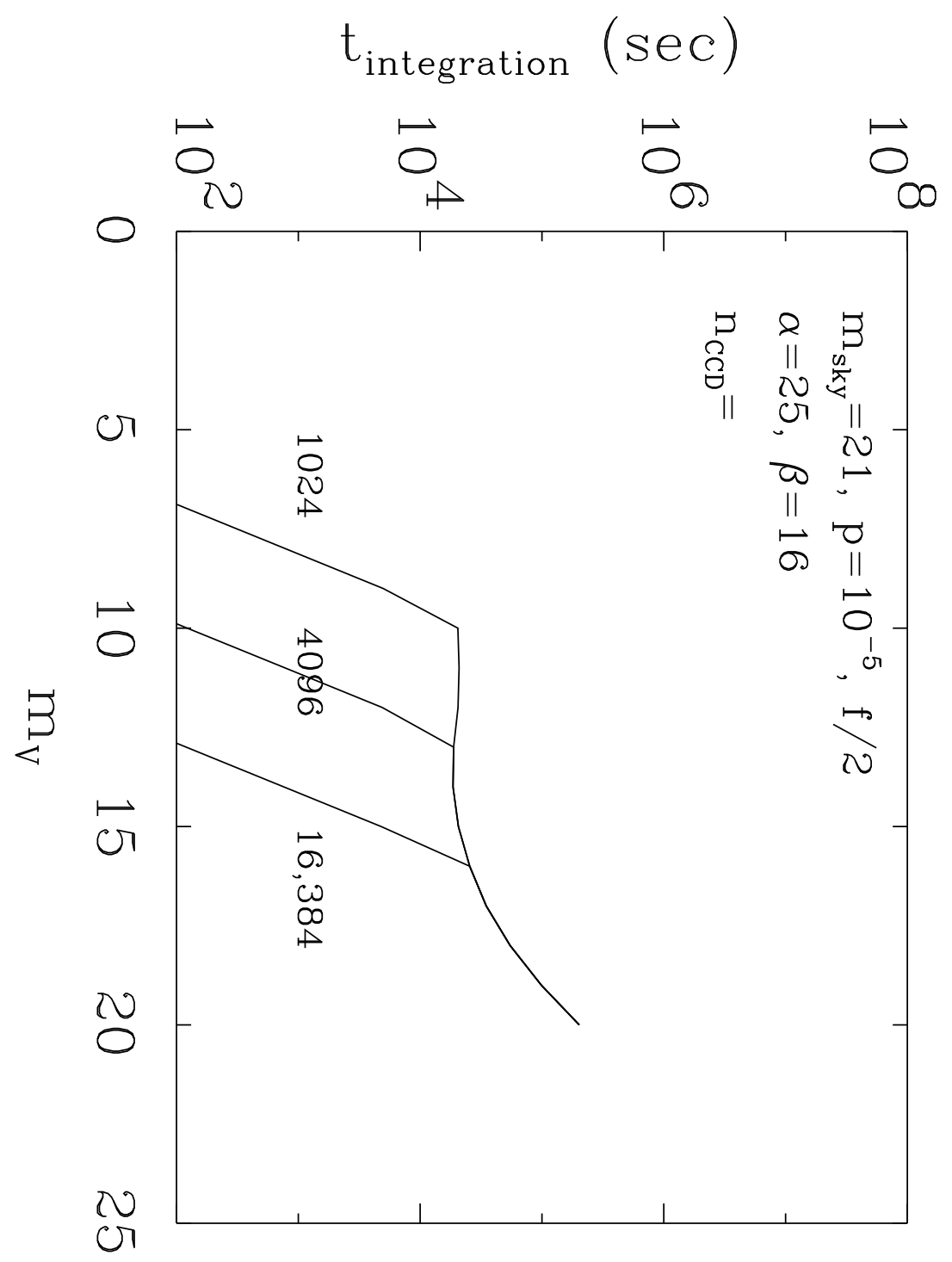




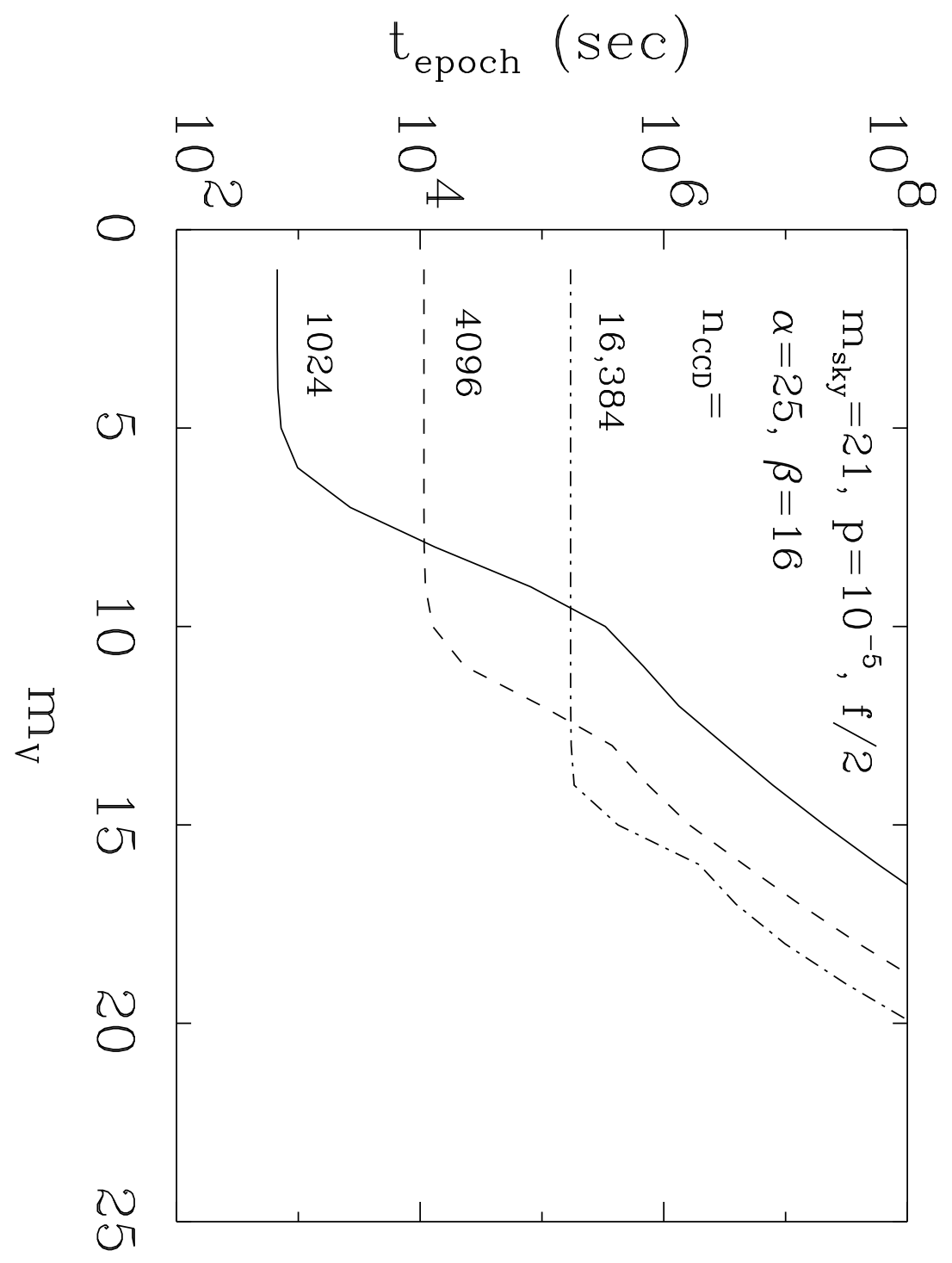




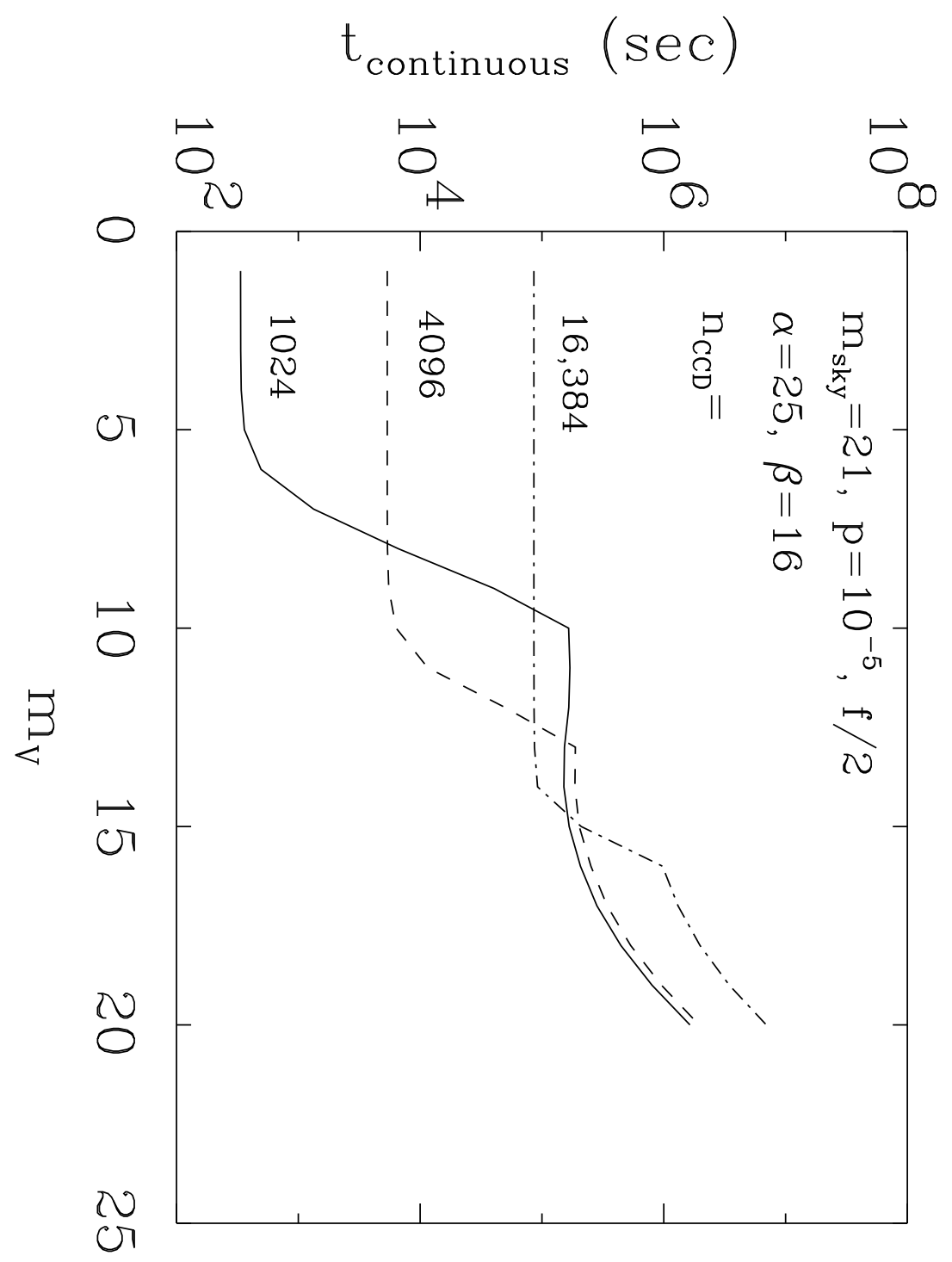




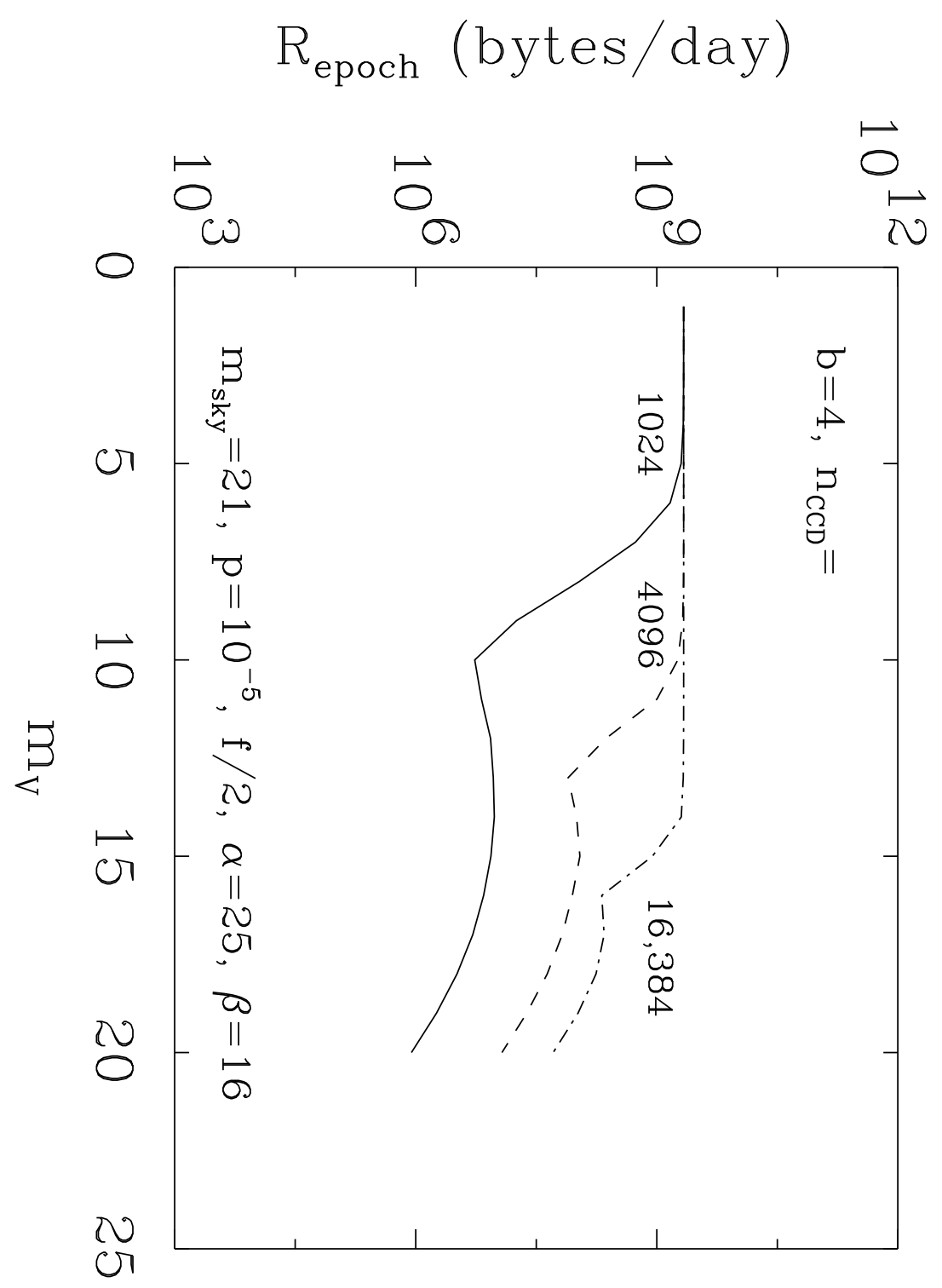




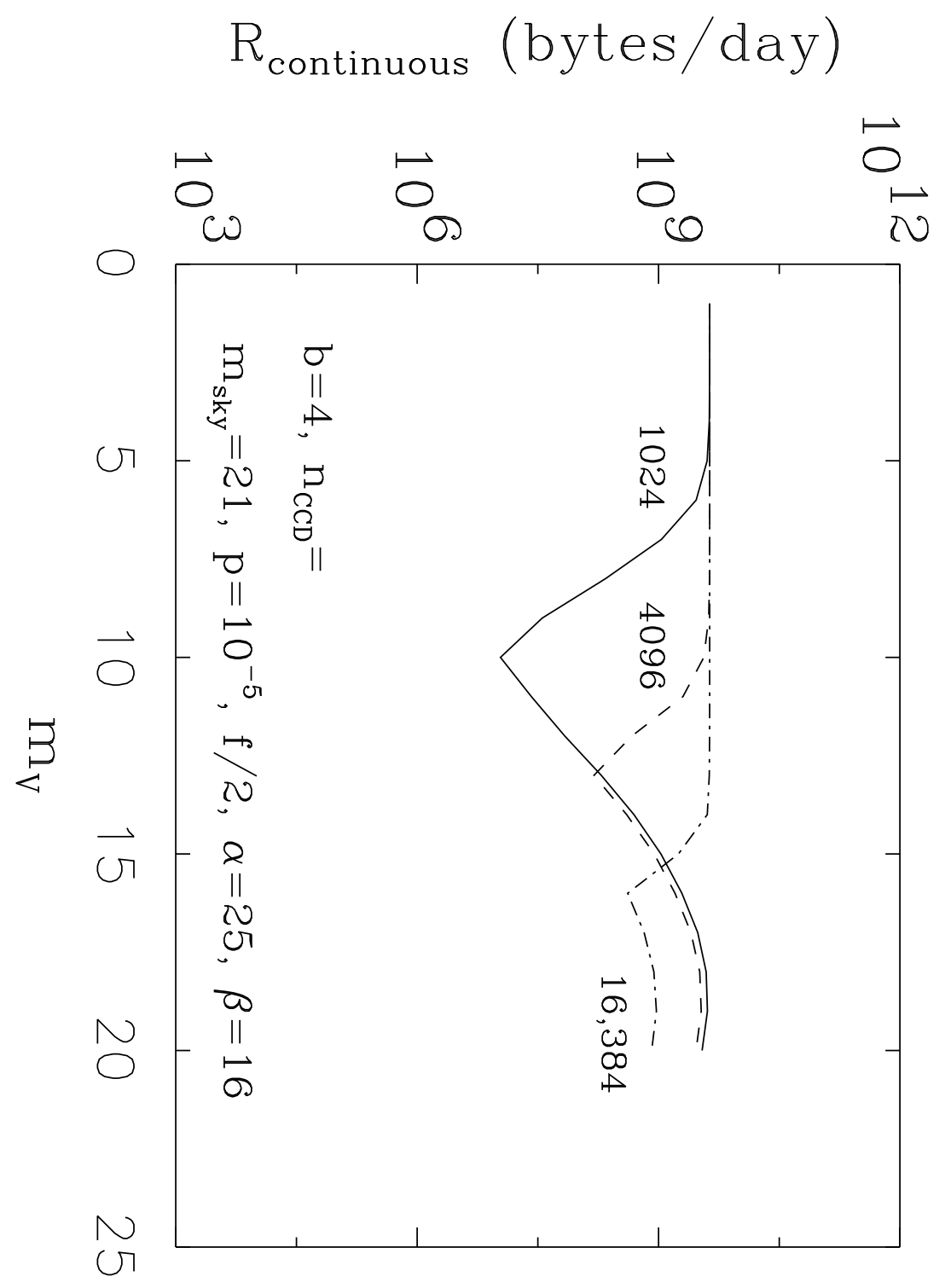


This figure "concampic.gif" is available in "gif" format from: http://arxiv.org/ps/astro-ph/9809403v2 
This figure "conskypic.gif" is available in "gif" format from: http://arxiv.org/ps/astro-ph/9809403v2 